\title{
INFINITE INTERVAL PROBLEMS ARISING IN THE MODEL OF A SLENDER DRY PATCH IN A LIQUID FILM DRAINING UNDER GRAVITY DOWN AN INCLINED PLANE *
}

\author{
RAVI P. AGARWAL ${ }^{\dagger}$ AND DONAL O'REGAN ${ }^{\ddagger}$
}

\begin{abstract}
Existence results are established for a second order boundary value problem on the half line motivated from the model of a slender dry patch in a liquid film draining under gravity down an inclined plane.
\end{abstract}

1. Introduction. Consider a thin film of viscous liquid with constant density $\rho$ and viscosity $\mu$ flowing down a planer substrate inclined at an angle $\alpha\left(0<\alpha \leq \frac{\pi}{2}\right)$ to the horizontal. We adopt Cartesian coordinates $(x, y, z)$ with the $x$-axis down the greatest slope and the $z$-axis normal to the plane. With the usual lubrication approximation the height of the free surface $z=h(x, y, z)$ satisfies [4]

$$
3 \mu h_{t}=\nabla \cdot\left[h^{3} \nabla\left(\rho g h \cos \alpha-\sigma \nabla^{2} h\right)\right]-\rho g \sin \alpha\left[h^{3}\right]_{x}
$$

where $t$ denotes time, $g$ the magnitude of acceleration due to gravity and $\sigma$ the coefficient of surface tension. We are interested in solutions symmetric about $y=0$, and we seek a steady state solution for a slender dry patch for which the length scale down the plane (i.e. in the $x$ direction) is much greater than in the transverse direction (i.e. in the $y$ direction), so the equation (1.1) is approximated by [4]

$$
\left[h^{3}\left(\rho g h \cos \alpha-\sigma h_{y y}\right)_{y}\right]_{y}-\rho g \sin \alpha\left[h^{3}\right]_{x}=0 .
$$

The velocity component down the plane is $u(x, y, z)=\frac{\rho g \sin \alpha\left[2 h z-z^{2}\right]}{2 \mu}$ and so for a slender dry patch of semi-width $y_{e}=y_{e}(x)$ the average volume flux around the dry patch per unit width in the transverse direction down the plane (denoted by $Q(x)$ ) is approximately [4]

$$
Q=\frac{\rho g \sin \alpha}{3 \mu} \lim _{y \rightarrow \infty} y^{-1} \int_{y_{e}(x)}^{y} h(x, w)^{3} d w .
$$

We seek a similarity solution to equation (1.2) of the form $h=f(x) G(\eta)$ where $\eta=\frac{y}{y_{e}(x)}$. Note $G(1)=0$ and (1.2) takes the form

$$
\begin{aligned}
& \rho g \cos \alpha f^{2} y_{e}^{2}\left(G^{3} G^{\prime}\right)^{\prime}-\sigma f^{2}\left(G^{3} G^{\prime \prime \prime}\right)^{\prime} \\
& \quad-3 \rho g \sin \alpha y_{e}^{3} G^{2}\left(f^{\prime} G y_{e}-f G^{\prime} y_{e}^{\prime} \eta\right)=0
\end{aligned}
$$

with the corresponding expression for $Q$ being

$$
Q=\frac{\rho g \sin \alpha}{3 \mu} f^{3} \lim _{\eta \rightarrow \infty} \eta^{-1} \int_{1}^{\eta} G(w)^{3} d w .
$$

For weak surface-tension effects the second term in (1.4) can be neglected and so the only relevant similarity solution is given (after a suitable choice of origin in $x$ ) by

$$
f(x)=b(c x)^{m} \text { and } y_{e}(x)=(c x)^{k}
$$

\footnotetext{
*Received March 31, 2003; accepted for publication August 21, 2003.

${ }^{\dagger}$ Department of Mathematical Sciences, Florida Institute of Technology, Melbourne, Florida 32901-6975, USA (agarwal@fit.edu).

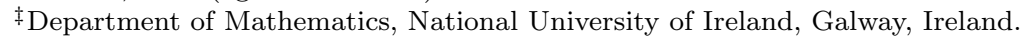


where the coefficients $b$ and $c$ and the exponents $m$ and $k$ are constants with $m=2 k-1$. In this case $\alpha \neq \frac{\pi}{2}$ and so we may choose without loss of generality $b=c k \tan \alpha$ and so (1.4) becomes

$$
\left(\left(G^{\prime}+\eta\right)^{\prime} G^{3}\right)^{\prime}-\left(7-\frac{3}{k}\right) G^{3}=0 .
$$

The unknown exponent $k$ is determined by the requirement that the average volume flux per unit width around the dry patch, $Q$, is independent of $x$. This is possible only if $m=0$ and $G \sim G_{0}>0$ (a constant) as $\eta \rightarrow \infty$. Thus

$$
Q=\frac{\rho g \sin \alpha}{3 \mu}\left(b G_{0}\right)^{3} \text { and so } m=0, k=\frac{1}{2} .
$$

Setting $k=\frac{1}{2}$ in (1.5) yields

$$
\left(G^{3} G^{\prime}\right)^{\prime}+\eta\left(G^{3}\right)^{\prime}=0 .
$$

Also the solutions to (1.6) must satisfy the boundary condition $G(1)=0$ and the far-field condition $\lim _{\eta \rightarrow \infty} G(\eta)=G_{0}$. As a result one is interested in the boundary value problem

$$
\left\{\begin{array}{l}
\left(G^{3} G^{\prime}\right)^{\prime}+\eta\left(G^{3}\right)^{\prime}=0, \quad 1<\eta<\infty \\
G(1)=0, \lim _{\eta \rightarrow \infty} G(\eta)=G_{0}>0 .
\end{array}\right.
$$

Keeping this problem in mind, in Section 2 we discuss the general boundary value problem

$$
\left\{\begin{array}{l}
\left(G^{\prime}(y)+p(t) y^{m}\right)^{\prime}+q(t) f(t, y)=p^{\prime}(t) y^{m}, a<t<n \\
y(a)=0, y(n)=b_{0}>0,
\end{array}\right.
$$

where $n>a, G(z)=\int_{0}^{z} g(x) d x, G^{\prime}(y)=\frac{d}{d t} G(y(t))$ and

$$
g(x)=\left\{\begin{array}{l}
x^{m}, x \geq 0 \\
-x^{m}, x<0
\end{array}\right.
$$

with $m>0$ odd. A very general existence theory will be presented for (1.8) in Section 2. Our theory relies on the following nonlinear alternative of Leray-Schauder type $[1$, 2].

TheOREM 1.1. Let $U$ be an open subset of a Banach space $E, J: \bar{U} \rightarrow E$ a continuous compact map, $p^{\star} \in U$ and let $N: \bar{U} \times[0,1] \rightarrow E$ be a continuous compact map with $N_{1}=J$ and $N_{0}=p^{\star}$ (here $N_{\lambda}(u)=N(u, \lambda)$ ). Also assume

$$
u \neq N_{\lambda}(u) \text { for } u \in \partial U \text { and } \lambda \in(0,1] .
$$

Then $J$ has a fixed point in $U$.

In Section 3 we discuss the following boundary value problem on the half line

$$
\left\{\begin{array}{l}
\left(G^{\prime}(y)+p(t) y^{m}\right)^{\prime}+q(t) f(t, y)=p^{\prime}(t) y^{m}, a<t<\infty \\
y(a)=0, y \text { bounded on }[a, \infty),
\end{array}\right.
$$

and our existence theory will then be applied to (1.7). 
2. Existence theory on finite intervals. In this section we first establish the existence of a solution to

$$
\left\{\begin{array}{l}
\left(G^{\prime}(y)+p(t) y^{m}\right)^{\prime}+q(t) f(t, y)=p^{\prime}(t) y^{m}, a<t<n \\
y(a)=0, y(n)=b_{0}>0
\end{array}\right.
$$

(here $n>a$ ) where $G(z)=\int_{0}^{z} g(x) d x$ and

$$
g(x)=\left\{\begin{array}{l}
x^{m}, x \geq 0 \\
-x^{m}=|x|^{m}, x<0
\end{array}\right.
$$

and with $m>0$ odd. Note $G^{\prime}(y)=\frac{d}{d t} G(y(t))$ and

$$
G(z)=\left\{\begin{array}{l}
\frac{z^{m+1}}{m+1}, \quad z \geq 0 \\
\frac{-z^{m+1}}{m+1}=\frac{-|z|^{m+1}}{m+1}, \quad z<0
\end{array}\right.
$$

By a solution to (2.1) we mean a function $y \in C[a, n]$, with $G(y) \in C^{1}[a, n], G^{\prime}(y)+$ $p y^{m} \in A C[a, n] \cap C^{1}(a, n]$ which satisfies $y(a)=0, y(n)=b_{0}$ and the differential equation in $(2.1)$ on $(a, n)$.

THEOREM 2.1. Suppose the following conditions are satisfied:

$$
\begin{gathered}
f:[a, n] \times \mathbf{R} \rightarrow \mathbf{R} \text { is continuous } \\
q \in C(a, n] \cap L^{1}[a, n] \text { with } q>0 \text { on }(a, n] \\
p \in C^{1}[a, n] \text { with } p \geq 0 \text { on }[a, n] \\
f(t, 0) \geq 0 \text { for } t \in(a, n)
\end{gathered}
$$

and

$$
f\left(t, b_{0}\right) \leq 0 \text { for } t \in(a, n) .
$$

Then (2.1) has a solution $y$ with $0 \leq y(t) \leq b_{0}$ for $t \in[a, n]$.

Proof. Consider the boundary value problem

$$
\left\{\begin{array}{l}
\left(G^{\prime}(y)+\lambda p y^{m}\right)^{\prime}=\lambda f^{\star}(t, y), \quad a<t<n \\
y(a)=0, y(n)=b_{0}>0,0<\lambda \leq 1
\end{array}\right.
$$

with

$$
f^{\star}(t, y)=\left\{\begin{array}{l}
-q(t) f(t, 0)+y, \quad y<0 \\
-q(t) f(t, y)+p^{\prime}(t) y^{m}, \quad 0 \leq y \leq b_{0} \\
-q(t) f\left(t, b_{0}\right)+p^{\prime}(t) b_{0}^{m}+y-b_{0}, \quad y>b_{0}
\end{array}\right.
$$

Solving $(2.7)_{\lambda}$ is equivalent (see [2]) to finding a $y \in C[a, n]$ which satisfies

$$
y(t)=G^{-1}\left(A(t-a)-\lambda \int_{a}^{t} p(s) y^{m}(s) d s+\lambda \int_{a}^{t}(t-x) f^{\star}(x, y(x)) d x\right)
$$


where

$$
A=\frac{G\left(b_{0}\right)+\lambda \int_{a}^{n} p(s) y^{m}(s) d s-\lambda \int_{a}^{n}(n-x) f^{\star}(x, y(x)) d x}{n-a} .
$$

Define the operator $N_{\lambda}: C[a, n] \rightarrow C[a, n]$ by

$$
N_{\lambda} y(t)=G^{-1}\left(A(t-a)-\lambda \int_{a}^{t} p(s) y^{m}(s) d s+\lambda \int_{a}^{t}(t-x) f^{\star}(x, y(x)) d x\right) .
$$

The argument in [2] guarantees that $N_{\lambda}: C[a, n] \rightarrow C[a, n]$ is continuous and completely continuous. We now show any solution $y$ to $(2.7)_{\lambda}(0<\lambda \leq 1)$ satisfies

$$
0 \leq G(y(t)) \leq G\left(b_{0}\right) \text { for } t \in[a, n]
$$

If $(2.10)$ is true then

$$
0 \leq y(t) \leq b_{0} \text { for } t \in[a, n]
$$

Suppose $G(y(t))<0$ for some $t \in(a, n)$. Then $G(y)$ has a negative minimum at say $t_{0} \in(a, n)$, so $G^{\prime}\left(y\left(t_{0}\right)\right)=0$. Also there exists $\delta_{1}>0, \delta_{2}>0$ with $\left(t_{0}-\delta_{1}, t_{0}+\delta_{2}\right) \subseteq$ $[a, n]$ and with

$$
\left\{\begin{array}{l}
G(y(t))<0 \text { for } t \in\left(t_{0}-\delta_{1}, t_{0}+\delta_{2}\right) \\
\text { and } G\left(y\left(t_{0}-\delta_{1}\right)\right)=G\left(y\left(t_{0}+\delta_{2}\right)\right)=0
\end{array}\right.
$$

Now for $t \in\left(t_{0}-\delta_{1}, t_{0}+\delta_{2}\right)$ we have

$$
\left(G^{\prime}(y(t))+\lambda p(t) y^{m}(t)\right)^{\prime}=-\lambda q(t) f(t, 0)+\lambda y(t)<0,
$$

so integration from $t_{0}$ to $t_{0}+\delta_{2}$ yields

$$
G^{\prime}\left(y\left(t_{0}+\delta_{2}\right)\right)+\lambda p\left(t_{0}+\delta_{2}\right) y^{m}\left(t_{0}+\delta_{2}\right)<\lambda p\left(t_{0}\right) y^{m}\left(t_{0}\right) .
$$

Now $y\left(t_{0}+\delta_{2}\right)=0$, so (note $m$ is odd, $p \geq 0$ and $y\left(t_{0}\right)<0$ )

$$
G^{\prime}\left(y\left(t_{0}+\delta_{2}\right)\right)<\lambda p\left(t_{0}\right) y^{m}\left(t_{0}\right) \leq 0 .
$$

Thus there exists $\delta_{3}>0, \delta_{3}<\delta_{2}$ with

$$
G^{\prime}(y(t))<0 \text { for } t \in\left(t_{0}+\delta_{3}, t_{0}+\delta_{2}\right) .
$$

As a result

$$
0=G\left(y\left(t_{0}+\delta_{2}\right)\right)<G\left(y\left(t_{0}+\delta_{3}\right)\right),
$$

and this contradicts (2.12). Thus $0 \leq G(y(t))$ for $t \in[a, n]$, so $0 \leq y(t)$ for $t \in[a, n]$. Next suppose $G(y(t))>G\left(b_{0}\right)$ for some $t \in(a, n)$. Then $G(y)$ has a positive maximum at say $t_{1} \in(a, n)$, so $G^{\prime}\left(y\left(t_{1}\right)\right)=0$. Also there exists $\delta_{4}>0, \delta_{5}>0$ with $\left(t_{1}-\delta_{4}, t_{1}+\delta_{5}\right) \subseteq[a, n]$ and with

$$
G(y(t))>G\left(b_{0}\right) \text { for } t \in\left(t_{1}-\delta_{4}, t_{1}+\delta_{5}\right)
$$

and

$$
G\left(y\left(t_{1}-\delta_{4}\right)\right)=G\left(y\left(t_{1}+\delta_{5}\right)\right)=G\left(b_{0}\right) .
$$


Also for $t \in\left(t_{1}-\delta_{4}, t_{1}+\delta_{5}\right)$ we have

$$
\begin{aligned}
\left(G^{\prime}(y(t))+\lambda p(t) y^{m}(t)\right)^{\prime} & =-\lambda q(t) f\left(t, b_{0}\right)+\lambda p^{\prime}(t) b_{0}^{m}+\lambda\left(y(t)-b_{0}\right) \\
& >\lambda p^{\prime}(t) b_{0}^{m},
\end{aligned}
$$

so integration from $t_{1}$ to $t_{1}+\delta_{5}$ yields (note $(2.16)$ )

$$
G^{\prime}\left(y\left(t_{1}+\delta_{5}\right)\right)+\lambda p\left(t_{1}+\delta_{5}\right) b_{0}^{m}>\lambda p\left(t_{1}\right) y^{m}\left(t_{1}\right)+\lambda b_{0}^{m}\left[p\left(t_{1}+\delta_{5}\right)-p\left(t_{1}\right)\right] .
$$

Thus

$$
G^{\prime}\left(y\left(t_{1}+\delta_{5}\right)\right)>\lambda p\left(t_{1}\right)\left[y^{m}\left(t_{1}\right)-b_{0}^{m}\right] \geq 0
$$

since $p \geq 0$. As a result there exists $\delta_{6}>0, \delta_{6}<\delta_{5}$ with

$$
G^{\prime}(y(t))>0 \text { for } t \in\left(t_{1}+\delta_{6}, t_{1}+\delta_{5}\right),
$$

$\mathrm{SO}$

$$
G\left(b_{0}\right)=G\left(y\left(t_{1}+\delta_{5}\right)\right)>G\left(y\left(t_{1}+\delta_{6}\right)\right),
$$

and this contradicts (2.15). Thus $G(y(t)) \leq G\left(b_{0}\right)$ for $t \in[a, n]$, so (2.11) holds.

Now Theorem 1.1 applied to $N_{\lambda}$ with $E=C[a, n], U=\{u \in E$ : $\left.\sup _{[a, n]}|u(t)|<b_{0}+1\right\}$ and $p^{\star}=G^{-1}\left(\frac{G\left(b_{0}\right)(t-a)}{n-a}\right)$ guarantees that $N_{1}$ has a fixed point $y \in U$. Thus $y$ is a solution of $(2.7)_{1}$ and the argument above guarantees that $0 \leq y(t) \leq b_{0}$ for $t \in[a, n]$. As a result $y$ is a solution of (2.1).

REMARK 2.1. It is possible to replace $p \geq 0$ on $[a, n]$ by $p \leq 0$ on $[a, n]$ and the result in Theorem 2.1 is again true; we leave the details to the reader.

Keeping our application in Section 1 in mind we now discuss the situation when our solution to $(2.1)$ is positive on $(a, n]$. Suppose the following conditions hold:

$$
\left\{\begin{array}{l}
\exists \alpha \in C[a, n] \text { with } G(\alpha) \in C^{1}[a, n], G^{\prime}(\alpha)+p \alpha^{m} \in A C[a, n] \\
\cap C^{1}(a, n] \text { with } b_{0} \geq \alpha>0 \text { on }(a, n], \alpha(a)=0, \alpha(n) \leq b_{0} \\
\text { and }\left(G^{\prime}(\alpha)+p \alpha^{m}\right)^{\prime}+q(t) f(t, \alpha) \geq p^{\prime}(t) \alpha^{m}(t) \text { on }(a, n)
\end{array}\right.
$$

$$
\left\{\begin{array}{l}
\text { for each } t \in(a, n) \text { we have } q(t)[f(t, y)-f(t, \alpha(t)] \geq 0 \\
\text { for } 0 \leq y \leq \alpha(t)
\end{array}\right.
$$

and

$$
p^{\prime}>0 \text { on }(a, n) .
$$

Also in this case we discuss the boundary value problem

$$
\left\{\begin{array}{l}
\left(y^{m} y^{\prime}\right)^{\prime}+p\left(y^{m}\right)^{\prime}+q f(t, y)=0, a<t<n \\
y(a)=0, y(n)=b_{0}>0 .
\end{array}\right.
$$

By a solution to (2.20) we mean a function $y \in C[a, n] \cap C^{1}(a, n]$ with $G(y) \in C^{1}[a, n]$, $y^{m} y^{\prime} \in C^{1}(a, n]$ which satisfies $y(a)=0, y(n)=b_{0}$ and the differential equation in $(2.20)$ on $(a, n)$. 
Theorem 2.2. Suppose (2.2)-(2.6), (2.17), (2.18) and (2.19) are satisfied. Then (2.1) has a solution $y$ with $\alpha(t) \leq y(t) \leq b_{0}$ for $t \in[a, n]$. In addition $y \in C^{1}(a, n]$ with $G^{\prime}(y)=y^{m} y^{\prime}$ on $(a, n)$ and $y$ is a solution of $(2.20)$.

Proof. Theorem 2.1 guarantees that (2.1) has a solution $y$ with $0 \leq y(t) \leq b_{0}$ for $t \in[a, n]$. Next we claim that

$$
y(t) \geq \alpha(t) \text { for } t \in[a, n] .
$$

Suppose $G(\alpha(t))>G(y(t))$ for some $t \in(a, n)$. Then $G(y)-G(\alpha)$ has a negative minimum at say $t_{0} \in(a, n)$, so $G^{\prime}\left(y\left(t_{0}\right)\right)=G^{\prime}\left(\alpha\left(t_{0}\right)\right)$. Also there exists $\delta_{1}>0, \delta_{2}>$ 0 with $\left(t_{0}-\delta_{1}, t_{0}+\delta_{2}\right) \subseteq[a, n]$ and with

$$
G(y(t))<G(\alpha(t)) \text { for } t \in\left(t_{0}-\delta_{1}, t_{0}+\delta_{2}\right)
$$

and

$$
G\left(y\left(t_{0}-\delta_{1}\right)\right)=G\left(\alpha\left(t_{0}-\delta_{1}\right)\right) \text { and } G\left(y\left(t_{0}+\delta_{2}\right)\right)=G\left(\alpha\left(t_{0}+\delta_{2}\right)\right) .
$$

Also for $t \in\left(t_{0}-\delta_{1}, t_{0}+\delta_{2}\right)$ we have (note $0 \leq y \leq b_{0}$ on $[a, n]$ )

$$
\begin{aligned}
\left(G^{\prime}(y)+p y^{m}\right)^{\prime}(t)-\left(G^{\prime}(\alpha)+p \alpha^{m}\right)^{\prime}(t) & \leq q(t)[f(t, \alpha(t))-f(t, y(t))] \\
& +p^{\prime}(t)\left[y^{m}(t)-\alpha^{m}(t)\right] \\
& <0
\end{aligned}
$$

since $p^{\prime}>0$ on $(a, n)$. Integrate from $t_{0}$ to $t_{0}+\delta_{2}$ to obtain

$$
\begin{aligned}
& G^{\prime}\left(y\left(t_{0}+\delta_{2}\right)\right)+p\left(t_{0}+\delta_{2}\right) y^{m}\left(t_{0}+\delta_{2}\right)-G^{\prime}\left(y\left(t_{0}\right)\right)-p\left(t_{0}\right) y^{m}\left(t_{0}\right) \\
& \quad<G^{\prime}\left(\alpha\left(t_{0}+\delta_{2}\right)\right)+p\left(t_{0}+\delta_{2}\right) \alpha^{m}\left(t_{0}+\delta_{2}\right)-G^{\prime}\left(\alpha\left(t_{0}\right)\right)-p\left(t_{0}\right) \alpha^{m}\left(t_{0}\right),
\end{aligned}
$$

so $($ note $(2.23))$

$$
G^{\prime}\left(y\left(t_{0}+\delta_{2}\right)\right)-G^{\prime}\left(\alpha\left(t_{0}+\delta_{2}\right)\right)<p\left(t_{0}\right)\left[y^{m}\left(t_{0}\right)-\alpha^{m}\left(t_{0}\right)\right] \leq 0,
$$

since $p \geq 0$ on $[a, n]$. Thus there exists $\delta_{3}>0, \delta_{3}<\delta_{2}$ with

$$
G^{\prime}(y(t))-G^{\prime}(\alpha(t))<0 \text { for } t \in\left(t_{0}+\delta_{3}, t_{0}+\delta_{2}\right)
$$

As a result

$$
0=G\left(y\left(t_{0}+\delta_{2}\right)\right)-G\left(\alpha\left(t_{0}+\delta_{2}\right)\right)<G\left(y\left(t_{0}+\delta_{3}\right)\right)-G\left(\alpha\left(t_{0}+\delta_{3}\right)\right),
$$

i.e.

$$
G\left(\alpha\left(t_{0}+\delta_{3}\right)\right)<G\left(y\left(t_{0}+\delta_{3}\right)\right),
$$

and this contradicts (2.22). Thus $G(\alpha(t)) \leq G(y(t))$ for $t \in[a, n]$, so $\alpha(t) \leq y(t)$ for $t \in[a, n]$ i.e $(2.21)$ is true.

In particular note $y(t)>0$ for $t \in(a, n]$. Also

$$
\begin{aligned}
\frac{y^{m+1}(t)}{m+1} & =A(t-a)-\int_{a}^{t} p(s) y^{m}(s) d s \\
& +\int_{a}^{t}(t-x)\left[-q(x) f(x, y(x))+p^{\prime}(x) y^{m}(x)\right] d x
\end{aligned}
$$


where $A$ is given in (2.9) with $\lambda=1$ and $f^{\star}(x, y(x))=-q(x) f(x, y(x))+$ $p^{\prime}(x) y^{m}(x)$. Since $y>0$ on $(a, n]$ we have $y^{\prime} \in C(a, n]$. Then the change of variables theorem [3 pp. 181] guarantees that $G^{\prime}(y)=g(y) y^{\prime}=y^{m} y^{\prime}$ on $(a, n)$. Also for $t \in(a, n)$ we have

$$
g(y) y^{\prime}=A-p y^{m}+\int_{a}^{t}\left[-q(x) f(x, y(x))+p^{\prime}(x) y^{m}(x)\right] d x,
$$

so $g(y) y^{\prime} \in C^{1}(a, n)$. Thus for $t \in(a, n)$ we have

$$
-q f(t, y)+p^{\prime} y^{m}=\left(g(y) y^{\prime}+p y^{m}\right)^{\prime}=\left(g(y) y^{\prime}\right)^{\prime}+\left(p y^{m}\right)^{\prime},
$$

so $y$ is a solution of $(2.20)$.

Suppose the following condition is satisfied:

$$
\left\{\begin{array}{l}
\exists \alpha \in C[a, n] \cap C^{1}(a, n] \text { with } G(\alpha) \in C^{1}[a, n], \\
\alpha^{m} \alpha^{\prime} \in C^{1}(a, n], b_{0} \geq \alpha>0 \text { on }(a, n], \alpha(a)=0, \alpha(n) \leq b_{0} \\
\text { and }\left(\alpha^{m} \alpha^{\prime}\right)^{\prime}+p\left(\alpha^{m}\right)^{\prime}+q(t) f(t, \alpha) \geq 0 \text { on }(a, n) .
\end{array}\right.
$$

Then we have the following theorem.

TheOREM 2.3. Suppose (2.2)-(2.6), (2.18), (2.19) and (2.24) are satisfied. Then (2.20) has a solution $y$ with $\alpha(t) \leq y(t) \leq b_{0}$ for $t \in[a, n]$.

Proof. Now the change of variables theorem [3 pp. 181] guarantees that $G^{\prime}(\alpha)=$ $g(\alpha) \alpha^{\prime}=\alpha^{m} \alpha^{\prime}$ on $(a, n)$, so for $t \in(a, n)$ we have

$$
\begin{aligned}
\left(G^{\prime}(\alpha)+p \alpha^{m}\right)^{\prime}+q f(t, \alpha) & =\left(\alpha^{m} \alpha^{\prime}+p \alpha^{m}\right)^{\prime}+q f(t, \alpha) \\
& =\left(\alpha^{m} \alpha^{\prime}\right)^{\prime}+\left(p \alpha^{m}\right)^{\prime}+q f(t, \alpha) \\
& \geq\left(p \alpha^{m}\right)^{\prime}-p\left(\alpha^{m}\right)^{\prime}=p^{\prime} \alpha^{m} .
\end{aligned}
$$

Thus (2.17) holds and the result follows from Theorem 2.2.

3. Existence theory on infinite intervals. In this section we first establish the existence of a solution to

$$
\left\{\begin{array}{l}
\left(G^{\prime}(y)+p(t) y^{m}\right)^{\prime}+q(t) f(t, y)=p^{\prime}(t) y^{m}, a<t<\infty \\
y(a)=0, y \text { bounded on }[a, \infty)
\end{array}\right.
$$

where $g$ and $G$ are as in Section 2 and $m>0$ is odd. By a solution to (3.1) we mean a function $y \in B C[a, \infty)$ (bounded continuous functions on $[0, \infty)$ ) with $G(y) \in C^{1}[a, \infty), G^{\prime}(y)+p y^{m} \in A C_{l o c}[a, \infty) \cap C^{1}(a, \infty)$ which satisfies $y(a)=0$ and the differential equation in $(3.1)$ on $(a, \infty)$.

Theorem 3.1. Suppose the following conditions are satisfied:

$$
\begin{gathered}
f:[a, \infty) \times \mathbf{R} \rightarrow \mathbf{R} \text { is continuous } \\
q \in C(a, \infty) \cap L_{l o c}^{1}[a, \infty) \text { with } q>0 \text { on }(a, \infty) \\
p \in C^{1}[a, \infty) \text { with } p \geq 0 \text { on }[a, \infty)
\end{gathered}
$$


and

$$
\left\{\begin{array}{l}
\exists \mu \in L_{l o c}^{1}[a, \infty) \text { with }|f(t, u)| \leq \mu(t) \\
\text { for a.e. } t \in[a, \infty) \text { and } u \in\left[0, b_{0}\right] .
\end{array}\right.
$$

Then (3.1) has a solution $y$ with $0 \leq y(t) \leq b_{0}$ for $t \in[a, \infty)$.

Proof. Fix $n \in N=\{1,2, \ldots\}$ with $n \geq a+1$ and consider the boundary value problem

$$
\left\{\begin{array}{l}
\left(G^{\prime}(y)+p(t) y^{m}\right)^{\prime}+q(t) f(t, y)=p^{\prime}(t) y^{m}, a<t<n \\
y(a)=0, y(n)=b_{0}>0 .
\end{array}\right.
$$

Theorem 3.1 guarantees that there exists a solution $y_{n}$ to (3.8) (i.e. $y_{n} \in C[a, n]$, with $\left.G\left(y_{n}\right) \in C^{1}[a, n], G^{\prime}\left(y_{n}\right)+p y_{n}^{m} \in A C[a, n] \cap C^{1}(a, n]\right)$ with $0 \leq y_{n}(t) \leq b_{0}$ for $t \in[a, n]$. We now claim that there exist constants $A_{1}$ and $A_{2}$ (independent of $n$ ) with

$$
\left|G^{\prime}\left(y_{n}(t)\right)\right| \leq A_{1}+A_{2} \int_{a}^{t}\left|p^{\prime}(s)\right| d s+\int_{a}^{t} \mu(s) d s \text { for } t \in[a, n] .
$$

The mean value theorem guarantees that there exists $\xi \in(a, a+1)$ with $G^{\prime}\left(y_{n}(\xi)\right)=$ $G\left(y_{n}(a+1)\right)-G(0)$, and so

$$
\left|G^{\prime}\left(y_{n}(\xi)\right)\right| \leq G\left(b_{0}\right) \equiv K_{0} .
$$

To prove (3.9) we consider first the case when $t \in[a, n]$ and $t>\xi$. Integrate (3.8) from $\xi$ to $t$ to obtain (note (3.7)),

$$
\begin{aligned}
\left|G^{\prime}\left(y_{n}(t)\right)\right| \leq & \left|G^{\prime}\left(y_{n}(\xi)\right)\right|+\left|p(t) y^{m}(t)-p(\xi) y^{m}(\xi)\right| \\
& +b_{0}^{m} \int_{\xi}^{t}\left|p^{\prime}(s)\right| d s+\int_{\xi}^{t} \mu(s) d s \\
\leq & K_{0}+|p(\xi)|\left|y^{m}(t)-y^{m}(\xi)\right|+|p(t)-p(\xi)| y^{m}(t) \\
& +b_{0}^{m} \int_{a}^{t}\left|p^{\prime}(s)\right| d s+\int_{a}^{t} \mu(s) d s \\
\leq & K_{0}+2 b_{0}^{m} \sup _{s \in[a, a+1]} p(s)+b_{0}^{m}\left|\int_{\xi}^{t} p^{\prime}(s) d s\right| \\
& +b_{0}^{m} \int_{a}^{t}\left|p^{\prime}(s)\right| d s+\int_{a}^{t} \mu(s) d s \\
\leq & K_{0}+2 b_{0}^{m} \sup _{s \in[a, a+1]} p(s)+2 b_{0}^{m} \int_{a}^{t}\left|p^{\prime}(s)\right| d s \\
& +\int_{a}^{t} \mu(s) d s,
\end{aligned}
$$


so (3.9) is true in this case. Next consider the case when $t<\xi$. Note in particular that $t<a+1$. Integrate the differential equation in (3.8) from $t$ to $\xi$ to obtain

$$
\begin{aligned}
\left|G^{\prime}\left(y_{n}(t)\right)\right| \leq & K_{0}+|p(\xi)|\left|y^{m}(t)-y^{m}(\xi)\right|+|p(t)-p(\xi)| y^{m}(t) \\
& +b_{0}^{m} \int_{t}^{\xi}\left|p^{\prime}(s)\right| d s+\int_{t}^{\xi} \mu(s) d s \\
\leq & K_{0}+2 b_{0}^{m} \sup _{s \in[a, a+1]} p(s)+2 b_{0}^{m} \int_{a}^{a+1}\left|p^{\prime}(s)\right| d s \\
& +\int_{a}^{a+1} \mu(s) d s,
\end{aligned}
$$

so (3.9) is again true.

Thus (3.9) is true in all cases, so for $t, s \in[a, n]$ with $s<t$ we have

$$
\begin{aligned}
\left|G\left(y_{n}(s)\right)-G\left(y_{n}(t)\right)\right| & =\left|\int_{s}^{t} G^{\prime}\left(y_{n}(x)\right) d x\right| \leq A_{1}|t-s| \\
& +A_{2} \int_{s}^{t} \int_{a}^{x}\left|p^{\prime}(z)\right| d z d x+\int_{s}^{t} \int_{a}^{x} \mu(x) d z d x .
\end{aligned}
$$

We can do this argument for each $k \in N$ with $k \geq n$. Define for $k \geq n$ an integer

$$
u_{k}(x)=\left\{\begin{array}{l}
y_{k}(x), x \in[a, k] \\
b_{0}, \quad x \in[k, \infty)
\end{array}\right.
$$

so

$$
G\left(u_{k}(x)\right)=\left\{\begin{array}{l}
G\left(y_{k}(x)\right), \quad x \in[a, k] \\
G\left(b_{0}\right), \quad x \in[k, \infty) .
\end{array}\right.
$$

It is easy to see that

$$
\begin{aligned}
\left|G\left(u_{k}(s)\right)-G\left(u_{k}(t)\right)\right| & \leq A_{1}|t-s|+A_{2}\left|\int_{s}^{t} \int_{a}^{x}\right| p^{\prime}(z)|d z d x| \\
& +\left|\int_{s}^{t} \int_{a}^{x} \mu(x) d z d x\right| \text { for } t, s \in[a, \infty) .
\end{aligned}
$$

Consider $\left\{u_{k}\right\}_{k=n}^{\infty}$. The Arzela-Ascoli theorem guarantees that there is a subsequence $N_{n}^{\star}$ of $\{n, n+1, \ldots\}$ and a function $G\left(z_{n}\right) \in C[a, n]$ with $G\left(u_{k}\right)$ converging uniformly on $[a, n]$ to $G\left(z_{n}\right)$ as $k \rightarrow \infty$ through $N_{n}^{\star}$. This together with the fact that $G^{-1}$ is continuous and $G\left(u_{k}(t)\right) \in\left[0, b_{0}\right]$ for $t \in[a, n]$ implies $u_{k}$ converges uniformly on $[a, n]$ to $z_{n}$ as $k \rightarrow \infty$ through $N_{n}^{\star}$. Note $0 \leq z_{n}(t) \leq b_{0}$ for $t \in[a, n]$. Let $N_{n}=N_{n}^{\star} \backslash\{n\}$. Also the Arzela-Ascoli theorem guarantees the existence of a subsequence $N_{n+1}^{\star}$ of $N_{n}$ and a function $G\left(z_{n+1}\right) \in C[a, n+1]$ with $G\left(u_{k}\right)$ converging uniformly on $[a, n+1]$ to $G\left(z_{n+1}\right)$ as $k \rightarrow \infty$ through $N_{n+1}^{\star}$, and so $u_{k}$ converges uniformly on $[a, n+1]$ to $z_{n+1}$ as $k \rightarrow \infty$ through $N_{n+1}^{\star}$. Note $0 \leq z_{n+1}(t) \leq b_{0}$ for $t \in[a, n+1]$ and $z_{n+1}=z_{n}$ on $[a, n]$ since $N_{n+1}^{\star} \subseteq N_{n}$. Let $N_{n+1}=N_{n+1}^{\star} \backslash\{n+1\}$. Proceed inductively to obtain for $m \in\{n+2, n+3, \ldots\}$ a subsequence $N_{m}^{\star}$ of $N_{m-1}$ and a function $z_{m} \in C[a, m]$ with $u_{k}$ converges uniformly on $[a, m]$ to $z_{m}$ as $k \rightarrow \infty$ through $N_{m}^{\star}$. Note $0 \leq z_{m}(t) \leq b_{0}$ for $t \in[a, m]$ and $z_{m}=z_{m-1}$ on $[a, m-1]$. Let $N_{m}=N_{m}^{\star} \backslash\{m\}$. 
Define a function $y$ as follows. Fix $x \in(a, \infty)$ and let $l \in\{n, n+1, \ldots\}$ with $x \leq l$. Then define $y(x)=z_{l}(x)$ so $y \in C[a, \infty)$ and $0 \leq y(t) \leq b_{0}$ on $[a, \infty)$. Also for $n \in N_{l}$ we have

$$
\begin{aligned}
G\left(u_{n}(x)\right) & =A_{l}(x-a)-\int_{a}^{x} p(s) u_{n}^{m}(s) d s \\
& +\int_{a}^{x}(x-s)\left[-q(s) f\left(s, u_{n}(s)\right)+p^{\prime}(s) u_{n}^{m}(s)\right] d s
\end{aligned}
$$

where

$$
\begin{aligned}
A_{l}(l-a) & =G\left(u_{n}(l)\right)+\int_{a}^{l} p(s) u_{n}^{m}(s) d s \\
& -\int_{a}^{l}(l-s)\left[-q(s) f\left(s, u_{n}(s)\right)+p^{\prime}(s) u_{n}^{m}(s)\right] d s .
\end{aligned}
$$

Let $n \rightarrow \infty$ through $N_{l}$ to obtain

$$
\begin{aligned}
G\left(z_{l}(x)\right) & =A_{l}^{\star}(x-a)-\int_{a}^{x} p(s) z_{l}^{m}(s) d s \\
& +\int_{a}^{x}(x-s)\left[-q(s) f\left(s, z_{l}(s)\right)+p^{\prime}(s) z_{l}^{m}(s)\right] d s
\end{aligned}
$$

where

$$
\begin{aligned}
A_{l}^{\star}(l-a) & =G\left(z_{l}(l)\right)+\int_{a}^{l} p(s) z_{l}^{m}(s) d s \\
& -\int_{a}^{l}(l-s)\left[-q(s) f\left(s, z_{l}(s)\right)+p^{\prime}(s) z_{l}^{m}(s)\right] d s .
\end{aligned}
$$

Thus

$$
\begin{aligned}
G(y(x)) & =A_{l}^{\star}(x-a)-\int_{a}^{x} p(s) y^{m}(s) d s \\
& +\int_{a}^{x}(x-s)\left[-q(s) f(s, y(s))+p^{\prime}(s) y^{m}(s)\right] d s
\end{aligned}
$$

where

$$
\begin{aligned}
A_{l}^{\star}(l-a) & =G(y(l))+\int_{a}^{l} p(s) y^{m}(s) d s \\
& -\int_{a}^{l}(l-s)\left[-q(s) f(s, y(s))+p^{\prime}(s) y^{m}(s)\right] d s .
\end{aligned}
$$

We can do this for each $x>a$ and so the above integral equation yields for each $l \in N$ and $t \in[a, l]$ that

$$
G^{\prime}(y(t))=-p(t) y^{m}(t)+A_{l}^{\star}+\int_{a}^{t}\left[-q(s) f(s, y(s))+p^{\prime}(s) y^{m}(s)\right] d s,
$$

so $G^{\prime} \in C^{1}[a, l], G^{\prime}(y)+p y^{m} \in A C[a, l] \cap C^{1}(a, l]$ and

$$
\left(G^{\prime}(y)+p y^{m}\right)^{\prime}(t)=-q(t) f(t, y(t))+p^{\prime}(t) y^{m}(t) \text { for } t \in[a, l] .
$$


Keeping the application in section 1 in mind it is important to discuss the situation when our solution to (3.1) is positive on $(a, \infty)$. Suppose the following conditions hold:

$$
\left\{\begin{array}{l}
\exists \alpha \in B C[a, \infty) \text { with } G(\alpha) \in C^{1}[a, \infty), G^{\prime}(\alpha)+p \alpha^{m} \\
\in A C_{l o c}[a, \infty) \cap C^{1}(a, \infty) \text { with } b_{0} \geq \alpha>0 \text { on }(a, \infty), \\
\alpha(a)=0 \text { and }\left(G^{\prime}(\alpha)+p \alpha^{m}\right)^{\prime}(t)+q(t) f(t, \alpha) \geq p^{\prime}(t) \alpha^{m}(t) \\
\text { on }(a, \infty)
\end{array}\right.
$$

$$
\left\{\begin{array}{l}
\text { for each } t \in[a, \infty) \text { we have } q(t)[f(t, y)-f(t, \alpha(t)] \geq 0 \\
\text { for } 0 \leq y \leq \alpha(t)
\end{array}\right.
$$

and

$$
p^{\prime}>0 \text { on }(a, \infty) .
$$

Also in this case we discuss the boundary value problem

$$
\left\{\begin{array}{l}
\left(g(y) y^{\prime}\right)^{\prime}+p\left(y^{m}\right)^{\prime}+q f(t, y)=0, a<t<\infty \\
y(a)=0, y \text { bounded on }[a, \infty) .
\end{array}\right.
$$

By a solution to (3.13) we mean a function $y \in B C[a, \infty) \cap C^{1}(a, \infty)$ with $y^{m} y^{\prime} \in$ $C^{1}(a, \infty)$ which satisfies $y(a)=0$ and the differential equation in $(3.13)$ on $(a, \infty)$.

Theorem 3.2. Suppose (3.2)-(3.7), (3.10), (3.11) and (3.12) hold. Then (3.1) has a solution $y$ with $0 \leq y(t) \leq b_{0}$ for $t \in[a, \infty)$. In addition $y \in C^{1}(a, \infty)$ with $G^{\prime}(y)=y^{m} y^{\prime}$ on $(a, \infty)$ and $y$ is a solution of (3.13).

Proof. Fix $n \in N=\{1,2, \ldots\}$ with $n \geq a+1$ and consider (3.8). Theorem 2.2 guarantees that there exists a solution $y_{n}$ to $(3.8)$ with $\alpha(t) \leq y_{n}(t) \leq b_{0}$ for $t \in[a, n]$. Essentially the same reasoning as in Theorem 3.1 guarantees that (3.1) has a solution $y \in B C[a, \infty)$ with $G(y) \in C^{1}[a, \infty), G^{\prime}(y)+p y^{m} \in A C_{l o c}[a, \infty) \cap C^{1}(a, \infty)$ and with $\alpha(t) \leq y(t) \leq b_{0}$ for $t \in[a, \infty)$. In particular note $y>0$ on $(a, \infty)$. Fix $l \in\{n, n+1, \ldots\}$ and consider $t \in[a, l]$. We know (see Theorem 3.1) that

$$
\begin{aligned}
\frac{y^{m+1}(t)}{m+1} & =A_{l}^{\star}(t-a)-\int_{a}^{t} p(s) y^{m}(s) d s \\
& +\int_{a}^{t}(t-s)\left[-q(s) f(s, y(s))+p^{\prime}(s) y^{m}(s)\right] d s
\end{aligned}
$$

where

$$
\begin{aligned}
A_{l}^{\star}(l-a) & =G(y(l))+\int_{a}^{l} p(s) y^{m}(s) d s \\
& -\int_{a}^{l}(l-s)\left[-q(s) f(s, y(s))+p^{\prime}(s) y^{m}(s)\right] d s,
\end{aligned}
$$

and since $y>0$ on $(a, l]$ we have $y^{\prime} \in C^{1}(a, l)$. Then [3 pp. 181] guarantees that $G^{\prime}(y)=g(y) y^{\prime}=y^{m} y^{\prime}$ on $(a, l)$. Also for $t \in(a, l)$ we have

$$
g(y) y^{\prime}=A_{l}^{\star}-p y^{m}+\int_{a}^{t}\left[-q(s) f(s, y(s))+p^{\prime}(s) y^{m}(s)\right] d s,
$$


so $g(y) y^{\prime} \in C^{1}(a, l)$. In addition for $t \in(a, l)$ we have

$$
-q f(t, y)+p^{\prime} y^{m}=\left(g(y) y^{\prime}+p y^{m}\right)^{\prime}=\left(g(y) y^{\prime}\right)^{\prime}+\left(p y^{m}\right)^{\prime} .
$$

We can do this for each $l \in N$, so $y$ is a solution of (3.13).

REMARK 3.1. If $\lim _{t \rightarrow \infty} \alpha(t)=b_{0}$ (here $b_{0}$ is as in (3.6)) then the solution $y$ to (3.1) (guaranteed from Theorem 3.2) is a solution of the boundary value problem

$$
\left\{\begin{array}{l}
\left(g(y) y^{\prime}\right)^{\prime}+p\left(y^{m}\right)^{\prime}+q f(t, y)=0, a<t<\infty \\
y(a)=0, \lim _{t \rightarrow \infty} y(t)=b_{0} .
\end{array}\right.
$$

Suppose the following condition is satisfied:

$$
\left\{\begin{array}{l}
\exists \alpha \in B C[a, \infty) \cap C^{1}(a, \infty) \text { with } G(\alpha) \in C^{1}[a, \infty), \\
\alpha^{m} \alpha^{\prime} \in C^{1}(a, \infty), b_{0} \geq \alpha>0 \text { on }(a, \infty), \alpha(a)=0 \\
\text { and }\left(\alpha^{m} \alpha^{\prime}\right)^{\prime}+p\left(\alpha^{m}\right)^{\prime}+q(t) f(t, \alpha) \geq 0 \text { on }(a, \infty) .
\end{array}\right.
$$

Then we have the following theorem.

Theorem 3.3. Suppose (3.2)-(3.7), (3.11), (3.12) and (3.15) hold. Then (3.13) has a solution $y$ with $\alpha(t) \leq y(t) \leq b_{0}$ for $t \in[a, \infty)$.

Proof. Now [3 pp. 181] guarantees that $G^{\prime}(\alpha)=g(\alpha) \alpha^{\prime}=\alpha^{m} \alpha^{\prime}$ on $(a, l)$ for each $l \in N$, so for $t \in(a, l)$ we have

$$
\begin{aligned}
\left(G^{\prime}(\alpha)+p \alpha^{m}\right)^{\prime}+q f(t, \alpha) & =\left(\alpha^{m} \alpha^{\prime}+p \alpha^{m}\right)^{\prime}+q f(t, \alpha) \\
& =\left(\alpha^{m} \alpha^{\prime}\right)^{\prime}+\left(p \alpha^{m}\right)^{\prime}+q f(t, \alpha) \\
& \geq\left(p \alpha^{m}\right)^{\prime}-p\left(\alpha^{m}\right)^{\prime}=p^{\prime} \alpha^{m} .
\end{aligned}
$$

Thus (3.10) holds and the result follows from Theorem 3.2.

REMARK 3.2. If $\lim _{t \rightarrow \infty} \alpha(t)=b_{0}$ (here $b_{0}$ is as in (3.6)) then the solution $y$ to (3.13) (guaranteed from Theorem 3.3) is a solution of (3.14).

Example. (Slender dry patch in a liquid film).

From Section 1 consider the boundary value problem

$$
\left\{\begin{array}{l}
\left(y^{3} y^{\prime}\right)^{\prime}+t\left(y^{3}\right)^{\prime}=0, \quad 1<t<\infty \\
y(1)=0, \lim _{t \rightarrow \infty} y(t)=G_{0}>0
\end{array}\right.
$$

We will now use Theorem 3.3 (with Remark 3.2) to show that (3.16) has a solution. To see this consider

$$
\left\{\begin{array}{l}
\left(y^{3} y^{\prime}+t y^{3}\right)^{\prime}=y^{3}, \quad 1<t<\infty \\
y(1)=0, y \text { bounded on }[1, \infty) .
\end{array}\right.
$$

REMARK 3.3. Notice $y \equiv 0$ is a solution of (3.17).

Let $m=3, a=1, p=t, q \equiv 0, f(t, y) \equiv 0, b_{0}=G_{0}$ and

$$
g(z)=\left\{\begin{array}{l}
z^{3}, z \geq 0 \\
-z^{3}=|z|^{3}, \quad z<0 .
\end{array}\right.
$$


Clearly (3.2)-(3.7), (3.11) and (3.12) hold. Let

$$
\alpha(t)=A \int_{1}^{t} \exp \left(-\frac{3 s^{2}}{2 G_{0}}\right) d s
$$

where

$$
A=\frac{G_{0}}{\int_{1}^{\infty} \exp \left(-\frac{3 s^{2}}{2 G_{0}}\right) d s} .
$$

Note $\alpha(1)=0$ and $\alpha^{\prime}=A \exp \left(-\frac{3 t^{2}}{2 G_{0}}\right)$. Also for $t \in(1, \infty)$ we have

$$
\begin{aligned}
\left(\alpha^{3} \alpha^{\prime}\right)^{\prime}+t\left(\alpha^{3}\right)^{\prime}= & A^{4}\left(\int_{1}^{t} \exp \left(-\frac{3 s^{2}}{2 G_{0}}\right) d s\right)^{2}\left[3 \exp \left(-\frac{3 t^{2}}{G_{0}}\right)\right. \\
& \left.-\frac{3 t}{G_{0}} \exp \left(-\frac{3 t^{2}}{2 G_{0}}\right) \int_{1}^{t} \exp \left(-\frac{3 s^{2}}{2 G_{0}}\right) d s\right] \\
& +3 t A^{3}\left(\int_{1}^{t} \exp \left(-\frac{3 s^{2}}{2 G_{0}}\right) d s\right)^{2} \exp \left(-\frac{3 t^{2}}{2 G_{0}}\right) \\
= & 3 t A^{3}\left(\int_{1}^{t} \exp \left(-\frac{3 s^{2}}{2 G_{0}}\right) d s\right)^{2} \exp \left(-\frac{3 t^{2}}{2 G_{0}}\right) \\
& \times\left[1-\frac{A}{G_{0}} \int_{1}^{t} \exp \left(-\frac{3 s^{2}}{2 G_{0}}\right) d s\right] \\
+ & 3 A^{4}\left(\int_{1}^{t} \exp \left(-\frac{3 s^{2}}{2 G_{0}}\right) d s\right)^{2} \exp \left(-\frac{3 t^{2}}{G_{0}}\right) \\
\geq & 0,
\end{aligned}
$$

since

$$
\frac{A}{G_{0}} \int_{1}^{t} \exp \left(-\frac{3 s^{2}}{2 G_{0}}\right) d s=\frac{\int_{1}^{t} \exp \left(-\frac{3 s^{2}}{2 G_{0}}\right) d s}{\int_{1}^{\infty} \exp \left(-\frac{3 s^{2}}{2 G_{0}}\right) d s} \leq 1 .
$$

Thus (3.15) holds so Theorem 3.3 guarantees that (3.17) has a solution $y$ with $\alpha(t) \leq$ $y(t) \leq G_{0}$ for $t \in[1, \infty)$. Also since $\lim _{t \rightarrow \infty} \alpha(t)=G_{0}$ then $y$ is a solution of (3.16).

\section{REFERENCES}

[1] D. O'REgAn, Existence theory for the equations $\left(G^{\prime}(y)\right)^{\prime}=q f\left(t, y, y^{\prime}\right)$ and $\left.\left(G^{\prime}(y)-p H(y)\right)\right]=$ $-p^{\prime} H(y)+q f(t, y)$, J. Math. Anal. Appl., 183 (1994), pp. 263-284.

[2] D. O'Regan, Boundary value problems on noncompact intervals, Proc. Edinburgh Math. Soc., 125A (1995), pp. 777-799.

[3] A. Torchinsky, Real Variables, Addison-Wesley, Redwood City, 1988.

[4] S.K. Wilson, B.R. Duffy AND S.H. DAVIS, On a slender dry patch in a liquid film draining under gravity down an inclined plane, Euro. Jnl. of Applied Mathematics, 12 (2001), pp. $233-252$. 
R. P. AGARWAL AND D. O'REGAN 